\title{
ASOCIACIÓN ENTRE EL TIEMPO DE ATENCIÓN PRE HOSPITALARIA Y LA MORTALIDAD HOSPITALARIA EN VÍCTIMAS DE ACCIDENTES DE TRÁNSITO
}

\author{
ASSOCIATION BETWEEN PRE-HOSPITAL CARE TIME AND HOSPITAL MORTALITY IN \\ VICTIMS OF TRAFFIC ACCIDENTS
}

María Jesús G. Fernandez-Sandoval', Bryam J. Vasquez-Zavala'

\begin{abstract}
RESUMEN
Introduccion: Los accidentes de tránsito (AT) se encuentran como la primera prioridad en investigación de salud en el Perú, Según la Organización Mundial de la Salud (OMS) se estima que los costos económicos por impacto de vehículos y lesiones por AT son de 1\% y 1,5\% del Producto Nacional Bruto (PNB) en países de bajos y medianos ingresos; En el Perú es aproximadamente el 1,5-2\% del Producto Bruto Interno (PBI). Objetivo: Determinar si existe asociación entre el tiempo de atención prehospitalaria y la mortalidad hospitalaria en víctimas de accidentes de tránsito. Métodos: Se realizó una revisión sistemática mediante la búsqueda bibliográfica de artículos relacionados en fuentes de indexación tales como PubMed y BIREME. Resultados: Los retrasos en las transferencias de pacientes al hospital y la falta Sistema Médico de Emergencia (SME) pre hospitalarios se asociaron significativamente con un aumento de la mortalidad (P: 0,000 ) así mismo la necesidad de un SME como proveedor de atención prehospitalaria fue predictor significativo de mortalidad en emergencia con un odds ratio [OR] ajustado 2,19 ; IC del $95 \%[1,88-2,55]$, y en las primeras 24 horas de ingreso con un OR ajustado 2,31; IC del 95\% [ 1,95-2,73]. Conclusión: EI tiempo de atención pre hospitalaria en accidentes de tránsito se encuentra significativamente asociada a la mortalidad hospitalaria, por ello, se propone estudiar factores asociados al largo tiempo de atención pre hospitalaria.
\end{abstract}

Palabras clave: Accidentes; Tráfico; Tiempo; Servicios médicos de emergencia; Atención de emergencia, prehospitalaria; Atención prehospitalaria de emergencia; Mortalidad (fuente: DeCS BIREME).

\begin{abstract}
Introduction: Traffic accidents (AT) are the first priority in health research in Peru, According to the World Health Organization (WHO) it is estimated that the economic costs of vehicle impact and injuries due to AT are $1 \%$ and $1.5 \%$ of the Gross National Product (GNP) in low and middle income countries; In Peru it is approximately 1.5 - 2\% of the Gross Domestic Product (GDP). Objective: To determine if there is an association between the time of prehospital care and hospital mortality in victims of traffic accidents. Methods: A systematic review was carried out through the literature search of related articles in indexing sources such as PubMed and BIREME. Results: Delays in the transfer of patients to the hospital and the lack of pre-hospital Emergency Medical System (SME) were significantly associated with an increase in mortality (P: 0.000$)$ and the need for an SME as a prehospital care provider was significant predictor of emergency mortality with an adjusted odds ratio [OR] 2.19;95\% Cl [1.88-2.55], and in the first 24 hours of admission with an adjusted OR $2.31 ; 95 \% \mathrm{Cl}$ [1.95-2.73]. Conclusion: The time of pre-hospital care in traffic accidents is significantly associated with hospital mortality, therefore, it is proposed to study factors associated with the longtime of pre-hospital care.
\end{abstract}

Key words: Accidents; Traffic; Disaster Victims; Time; Emergency Medical Services; Emergency Care, Prehospital; Prehospital Emergency Care; Mortality (source: MeSH NLM).

${ }^{1}$ Instituto de Investigación en Ciencias Biomédicas, INICIB, Universidad Ricardo Palma, Lima-Perú.

Citar como: María Jesús G. Fernandez-Sandoval, Bryam J. Vasquez-Zavala. Asociación entre el tiempo de atención pre hospitalaria y la mortalidad hospitalaria en víctimas de accidentes de tránsito. Rev. Fac. Med. Hum. Enero 2020; 20(1):144-152. DOI 10.25176/RFMH.v20i1.2558 


\section{INTRODUCCIÓN}

Actualmente los accidentes de tránsito (AT) se encuentran como la primera prioridad en investigación de salud en el Perú, debido a la gran mortalidad que este representa así como la gran cantidad de afectados que quedan con lesiones irreversibles. Esto ocasiona un elevado costo socioeconómico tanto por rehabilitación como la atención de las lesiones propiamente dichas.

Según la Organización Mundial de la Salud (OMS) cada día mueren en el mundo 3000 personas por AT, en especial en países de bajos y medianos ingresos. Se estima que al año en el mundo fallecen 1.2 millones de personas por accidentes en vía pública y 50 millones resultan heridos y que si no se mejoran y fortalecen los sistemas de prevención y reducción de impacto, aumentaría en un 65\% en 20 años $^{(1)}$.

La OMS estima que los costos económicos por impacto de vehículos y lesiones por AT son de $1 \%$ y $1.5 \%$ del Producto Nacional Bruto (PNB) en países de bajos y medianos ingresos respectivamente y que el costo mundial anual es cercano a US\$ 518000 millones ${ }^{(1)}$.

En el Perú, según el Consejo Nacional de Seguridad Vial (CNSV), cada año se registran aproximadamente $70 \mathrm{mil}$ de accidentes de tránsito con más de 3000 fallecidos y más de 45 mil heridos, de los cuales cientos de ellos desarrollan algún tipo de discapacidad permanente ${ }^{(2)}$.

Así mismo los costos los costos por accidentes de tránsito en el Perú podrían ascender a mil millones de dólares por año, aproximadamente el $1.5-2 \%$ del PBI, según la oficina general de estadística e informática y el instituto nacional de rehabilitación integrantes de la Estrategia Sanitaria Nacional de Accidentes de Tránsito (ESNAT) del ministerio de Salud ${ }^{(3)}$.

La respuesta a accidentes de tránsito es mediante los servicios médicos de emergencia, en el Perú los principales encargados de ello son el SAMU y los bomberos, entre otros entes de respuesta a emergencias, de su pronta y oportuna respuesta dependen supervivencia de la población afectada. El tiempo de atención pre hospitalaria se divide en cuatro partes, la primera en relación a la llamada del lugar del accidente y la partida del servicio asistencial, la segunda entre la salida del servicio asistencial y la llegada al accidente, la tercera el tiempo de asistencia en el mismo lugar del accidente y la cuarta desde el punto del accidente hasta la llegada al hospital para el oportuno manejo hospitalario del paciente(4).

Se plantea que una rápida atención pre hospitalaria estaría asociada con la disminución de la mortalidad debido a una oportuna y eficaz atención hospitalaria. El objetivo del estudio es conocer si existe asociación entre el tiempo de atención pre hospitalario y la mortalidad hospitalaria en víctimas de accidentes de tránsito.

\section{MÉTODOS}

Se planteó la pregunta ¿Cuál es la asociación entre el tiempo de atención pre hospitalaria y la mortalidad hospitalaria en víctimas de accidentes vehiculares? Y la pregunta PEO es: P: víctimas de accidentes de tránsito; E: amplio tiempo de atención pre hospitalaria; O: mortalidad. Para ello se realizó una revisión sistemática mediante la búsqueda bibliográfica de artículos relacionados en fuentes de indexación tales como PubMed y BIREME.

Los términos utilizados para la búsqueda en Pubmed fueron: Accidents, Traffic; Traffic Accidents; Accident, Traffic; Traffic Accident; Time; Longterm Effect; Emergency Medical Services; Prehospital Emergency Care; Emergency Care, Prehospital; Mortality; Case Fatality Rates; Excess Mortality; Mortality Decline; Mortality Determinants; Differential Mortality; Death Rate; Mortality Rate. Para ello se utilizó la sintaxis: ((((()"Accidents, Traffic"[mh]) OR Traffic Accidents*[tiab]) OR Accident, Traffic*[tiab]) OR Traffic Accident*[tiab])) AND ((((("Time"[mh]) OR Longterm Effect*[tiab]) AND "Emergency Medical Services"[mh]) OR Prehospital Emergency Care*[tiab]) OR Emergency Care, Prehospital*[tiab])) AND ((()(((("Mortality"[mh]) OR Case Fatality Rates*[tiab]) OR Excess Mortality*[tiab]) OR Mortality Decline*[tiab]) OR Mortality Determinants*[tiab]) OR Differential Mortality*[tiab]) OR Death Rate*[tiab]) OR Mortality Rate*[tiab]), así mismo se usaron otros términos como "Prehospital time association". Para la búsqueda en BIREME se usaron los términos: Emergency Care, Prehospital; Traffic Accident y mortality, siendo la sintaxis: (tw:(Emergency Care, Prehospital)) AND (tw:(Traffic Accident)) AND (tw:(mortality))

Se creó una base de datos a partir del programa de EXCEL.2013, donde se tabularon los resultados de la revisión en tablas.

\section{Criterios de inclusión:}

- Estudios con una antigüedad máxima de 10 años.

- Estudios con tiempo de atención pre hospitalaria en relación a accidentes de tránsito.

- Accidentes por colisión de vehículos motorizados.

- Estudios con información de tiempos pre hospitalarios. 
- Estudios cuya población sean traumatismos mayores y/o poli traumatizados.

\section{Criterios de Exclusión:}

- Estudios que se repitan en la búsqueda sistemática.

- Estudios de asociación de mortalidad con tiempo de atención pre hospitalaria por otra causa que no sea por accidentes de tránsito.

- Estudios que no muestren datos de asociación entre tiempo de atención pre hospitalaria y mortalidad hospitalaria

\section{RESULTADOS}

Se utilizaron 2 motores de búsqueda, ambos con distinta cantidad de estudios encontrados en relación a la búsqueda sistemática, en Pubmed, con la sintaxis antes mencionada y los criterios de exclusión se encontró 35 estudios, de los cuales solo 4 eran útiles para nuestra revisión, así mismo se utilizó otros términos como "prehospital time association" de donde se hallaron 6 estudios que contenían datos relevantes para nuestra revisión. En el buscador BIREME, con la sintaxis y los criterios de exclusión antes presentados se encontraron 134 artículos de estos, 3 contenían la información requerida para el presente estudio. La exclusión de los demás estudios en esta búsqueda sistemática se debió a la presencia de otras patologías, no asociadas a accidentes automovilísticos ni colisiones de vehículos motorizados, cuyas mortalidades estaban asociadas al tiempo de atención prehospitalario. De estos 13 estudios 2 son prospectivos y 11 son retrospectivos.

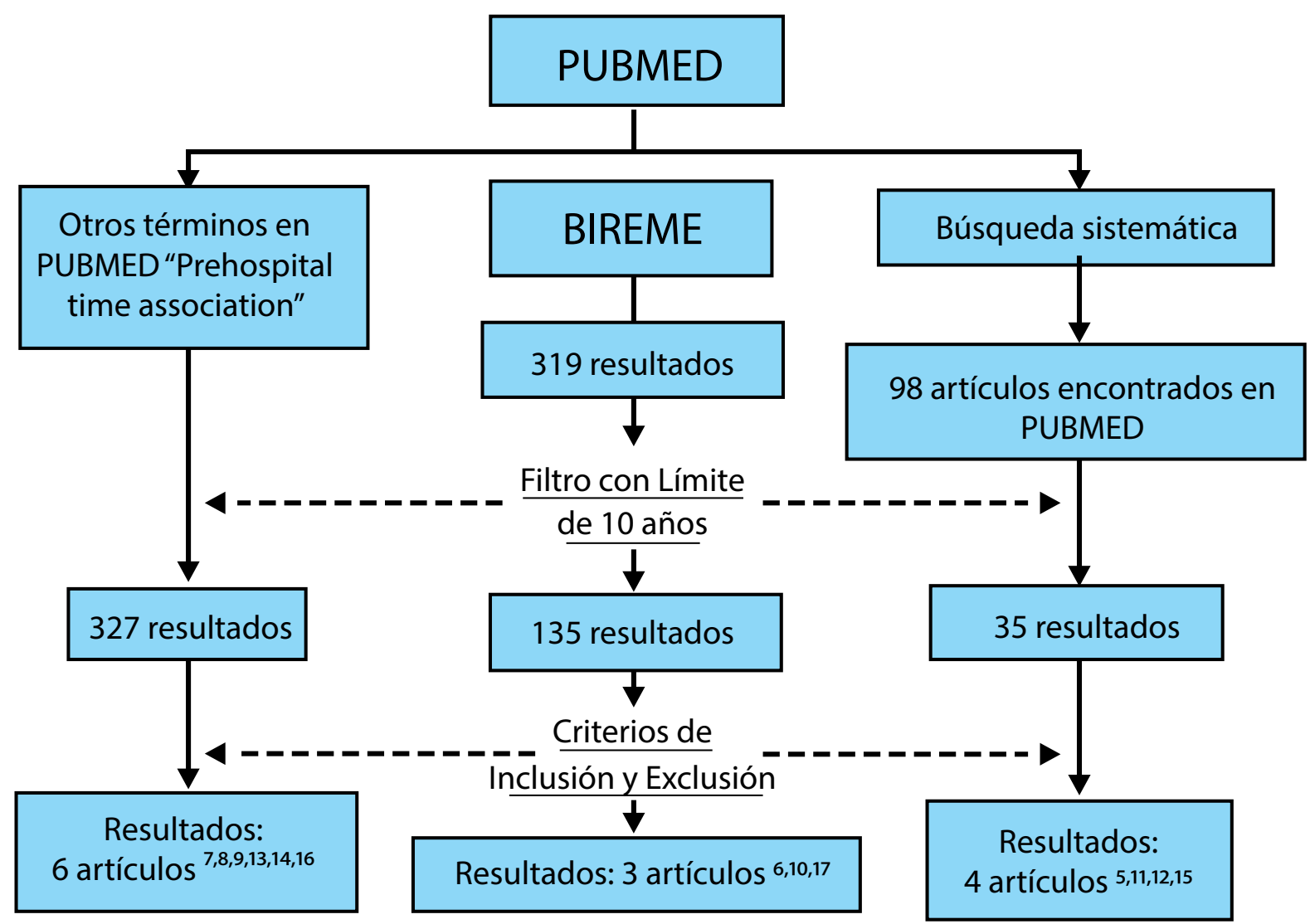

Figura 1. Diagrama de flujo de selección de artículos. 
Tabla 1. Características de los estudios de la revisión sistemática.

\begin{tabular}{|c|c|c|c|}
\hline Autores & Población & País & Tipo de estudio \\
\hline TOHME et al. ${ }^{5}$ & 589 & Suiza & Estudio de cohorte prospectivo \\
\hline Chandrasekharan et al. ${ }^{6}$ & 773 & India & Prospectivo observacional \\
\hline MILLS et al. ${ }^{7}$ & 18709 & Dinamarca & Cohorte retrospectivo \\
\hline BROWN et al. ${ }^{8}$ & 1625 & Australia & Cohorte retrospectivo \\
\hline BYRNE et al. ${ }^{9}$ & 119740 & EE.UU & Cohorte retrospectivo \\
\hline GONZALEZ et al. ${ }^{10}$ & 45,763 & EE.UU & Estudio retrospectivo \\
\hline LOVELY et al. ${ }^{11}$ & 3980 & EE.UU & Estudio retrospectivo \\
\hline RIYAPAN et al. ${ }^{12}$ & 39.761 & Tailandia & Cohorte retrospectivo \\
\hline GAUSS et al. ${ }^{13}$ & 6441 & Francia & Cohorte retrospectivo \\
\hline VERA-LOPEZ et al. ${ }^{14}$ & 2,575 & México & Estudio de casos y controles \\
\hline TANSLEY et al. ${ }^{15}$ & 1568 & Canadá & Estudio observacional analítico \\
\hline CHAMPION et al. ${ }^{16}$ & 55537 & EE.UU & Estudio observacional analítico \\
\hline BONIFACE et al. ${ }^{17}$ & 9316 & Tanzania & Estudio transversal \\
\hline
\end{tabular}

\section{Estudios prospectivos}

En el estudio de Chandrasekharan $A$ et al. ${ }^{(6)}$ la mortalidad con un tiempo de atención pre hospitalaria menor a una hora, de los 69 pacientes transportados fue de 7, cuyo valor es menor que en un intervalo de 1 a 6 horas, donde de los 197 pacientes fallecieron 44, lo mismo ocurre con el intervalo de 6 a 24 horas, en carreteras alejadas al centro de salud, donde de los 416 pacientes fallecieron 96 , la mortalidad fue mayor que los supervivientes en un tiempo de atención pre hospitalaria mayor a 24 horas donde de las 91 víctimas, 31 sobrevivieron y 60 fallecieron. Se encontró entonces que el retraso mayor de 24 horas se asoció significativamente a una mayor mortalidad $(P=0,00)$. A diferencia del estudio de cohorte prospectivo de TOHME et al.(5) se encontró que en mecanismos de trauma como los AT, caídas y otros mecanismos, el tiempo como factor de riesgo pre hospitalario de mortalidad es poco significativo $(P=0,264)$, no obstante la hipotensión $(P=0,009)$, la hipoxemia $(P=<0,0001)$ y la hipotermia $(P=<0,0001)$ fueron factores de riesgo pre hospitalario significativamente asociados a la mortalidad y alteración de la conciencia después de una lesión cerebral traumática grave.

\section{Estudios Retrospectivos}

En el trabajo de Mills et al.(7) de 18709 pacientes estudiados, solo 1687 eran víctimas de accidentes de tránsito, se encontró que el tiempo total prehospitalario, en áreas urbanas, de 0 a 30 minutos versus $>60$ minutos no se halla asociada de forma significativa ni a la mortalidad hospitalaria a 1 día ni a 30 días, al igual que en el análisis de Lovely et al. ${ }^{(11)}$ que halló que el tiempo en la escena del accidente y el tiempo de transporte no se asoció significativamente con la mortalidad ( $P=$ 0,31 y $\mathrm{P}=0,458$ ), así como en el estudio de Brown et al8 donde tampoco hubo asociación entre un tiempo pre hospitalario de $\geq 60$ minutos y una estadía hospitalaria más prolongada, sin embargo, aquí los sobrevivientes de 30 días, un minuto de retraso en el tiempo en la escena del incidente se asoció con 1,16 veces (IC del $95 \%$ 1,03-1,31) a una mayor estadía en el hospital. Todas las interacciones fueron significativas $(p>0,05)$.

En el estudio de En Gonzalez et al. ${ }^{(10)}$ y Gauss et al ${ }^{(13)}$ se mostró asociación con el aumento significativo y progresivo de la mortalidad con el tiempo total pre hospitalario, también se encontró que al aumento del tiempo pre hospitalario mayor a 30 minutos se asoció con un gran aumento en el riesgo de muerte en el hospital así como la demora mayor a 180 minutos. Así 
Prehospital time and morPrients requiring

2019 a highest priority emergency medical response:
a Danish registry-based cohort study.

\section{días no se asoció con el $\quad$ OR 0.65 (IC 95\% 0.29 a tiempo prehospitalario en 1.48 )} accidentes de tránsito.
Estudia la influencia del total, en la mortalidad de día como en la de 30 días

\section{Logró encontrar que} $\begin{array}{ll}\text { el tiempo prehospi- } & \text { Los hallazgos significativos } \\ \text { talario no afecta a la } & \text { son meramente asociaciones }\end{array}$ mortalidad hasta por y no causales. 80 minutos.
Association of Prehospital Time to In-Hospital Traum 2019 Mortality in a Physician-Staffed Emergency Medicine System

Longer Prehospital Tim was not Associated with

2019 Mortality in Major Trauma: A Retrospective Cohort Study.

Injury Severity Score alone predicts mortality when compared to EMS scen compard to EMS scene time and transport time for motor vehicle trauma patients who arrive alive to hospital

Effect of predicted trave time to trauma care on mortality in major trauma patients in Nova Scotia.

La falta de TTPH a centros de atención en 30 minutos (OR 1.66, intervalo de $\begin{array}{ll}\text { estaba asociado con un } & \text { confianza }[\mathrm{IC}] \text { del } 95 \% \\ 1.09-2.52, \mathrm{p}=0.018\end{array}$ $66 \%$ más de riesgo de muerte

asociación entre la oportunidad de intervención $\quad$ OR 0.99;IC95\%(0,97-1,01)

a respuesta del Sistema Médico de Emergencias y su relación con distintos

2018 resultados en salud en personas lesionadas por e tránsito de dos ciudades mexicanas.

Las probabilidades de

muerte aumentaron en un (OR, 1.09 [IC 95\%, 1.07-

[IC 95\%, 1.01-1.07]). (OR ajustado 1.10,95\% confidence interval (CI) talidad en 30 días. Pero en los sobrevivientes de 30 días, el aumento de un escena se asoció con 1.16 veces mayor duración de respectivamente. $\begin{array}{lll}\text { VERA-LOPEZ et al. }{ }^{(14)} & \text { (TAPH) con mortalidad y } & \mathrm{p}=0.318 \text { y OR 28.30; } \\ & \text { la atención prehospitalaria } & \text { IC } 95 \%(3,60-222,58) ;\end{array}$ asociada a consecuencias $\mathrm{p}=0.001$ negativas en salud a asociación entre el tiem o de atención prehospita ario (TAPH) y mortalidad.

El tiempo pre hos-

Los datos comparativos

variable de exposición existentes son limitados conflictivos.

Redujo el riesgo de potencial sesgo de sobrevi1.16 (IC del 95\%: 1.03-1.31) hospitalaria. ayor tiempo de estadía

El TAPH larga se asoció con peores resultados para víctimas por AT.
Se predijo significativamente la mortalidad.

Gran cantidad de escenas con TAPH prolongado.
No se pudo capturar las muertes pre hospitalarias.

Confiabilidad que

evitó la inclusión de Es posible la confusión análisis. datos inexactos en el residual por factores no
La oportunidad de la ción para el estudio.
El tamaño de la muestra para los casos de discapacidad y 
Outcomes of Emergency Medical Service Usage in Severe Road Traffic Injury during Thai Holidays
Uso del Sistema médico de emergencia como proveedor de a proveedor de atencior prehospitalaria fue predictor significativo de mortalidad en eme $y$ a las 24 horas.
[OR] ajustado 2.19; IC

del $95 \%$ [1.88-2.55], y en las primeras 24 horas de ingreso OR ajustado 2.31; IC del 95\% [ 1.95-2.73].

a asociación entre sistem de atención pre hospitalario, y la mortalidad.

e analizó el registro más grande del país en AT.
No recopiló datos sobre variables de confusión que podrían afectar los resultados clínicos.
Factors Impacting Mortality in the Pre-Hospital Period After Road Traffic Accidents in Urban India. ospital times on traum hospital times on trauma chmarking: An ecologic study.

Time and place of death from automobile crashes:

2016 Research endpointimplications

Prehospital risk factors of mortality and impaired

2014 consciousness after severe traumatic brain injury: an epidemiological study

INJURY EXPERIENCE IN ZANIA- NEED FOR INTERVENTION.

Los retrasos en las transferencias de pacientes al hospital y la falta de

Chandrasekharan A servicios de emergencia et al. ${ }^{(6)} \quad$ prehospitalarios se asociaron significativamente con un aumento de la mortalidad

Las probabilidades de

muerte con los PHT más

cortos fueron significativa- (OR, 2,00; intervalo de

mente confianza [IC] del $95 \%$

centros con los PHT más $\quad 1.43-2.78$ )

largos.

El riesgo de mortalidad

después del accidente es La curva resultante $Y$

CHAMPIONn et al. ${ }^{(16)}$ en los primeros 30 minuto en los próximos $60 \quad \mathrm{RR}=0.96$

la mortalidad. minutos.

El tiempo prehospitalario no se identificó como factor de riesgo o protector

$P=0.264$

La llegada tardía al hospital tuvo un impacto significativo en la mortalidad.

Contiene los datos necesarios para la asociación con la morbimortalidad.

a mortalidad es un resultado afectado por varias variables. Intrahospitalarias.

TOHME et al..$^{(3)}$ factores de mortalidad.

Uno de los factores asociados a mortalidad

fue el tiempo de atención

desde el accidente hasta

$P=0.000$

la llegada al hospital de

2 - 10 horas.

Does increased emer-

gency medical services

prehospital time affect

2009 prehospital time afect

patient mortality in rura

statewide analysis
Se asoció una mayor mor-

talidad en un tiempo de

GONZALES et al..$^{(10)}$

respuesta mayor en zonas

$P=0.0001$
Excelente selección la mortalidad ajustada. y calibración en los riesgo.

No se pueden sacar concausal.

Evaluar si existe oportunipor accidentes de tránsito.

establece la relación entre el tiempo des-

pués de la lesior muerte

lestudio se limitó a pacientes que sufrieron la lesion antes de las 4 horas y despues de los 5 minutos.

Hubo asociación entre hipo- Los resultados tuvie- Los resultados son asociaciotermia e hipotensión con la ron pocas pérdidas en nes y, por lo tanto, pueden mortalidad a corto plazo. el seguimiento. no ser causales.

Factores asociados con la mortalidad en seis hospitales de Tanzania.

Se encontró que los AT son la causa más tancia de los AT propiamente en dicho país.

Busca asociación entre e

Hace énfasis en la en relación a la mortalidad.

(n) énfasis

No toma en cuenta otras variables que puede presenta el paciente dad de reducir la mortalid 
mismo Tansley et al $^{(15)}$ se descubrió que demora mayor de 30 minutos TTPH se asociaba con un aumento del $66 \%$ en el riesgo de muerte después del ajuste por las variables de confusión de edad, sexo, escala de severidad de lesión identificadas a priori (odds ratio [OR] 1.66, intervalo de confianza [IC] del 95\%: $1,09-2.52, p=0,018)$. Sin embargo esta asociación se perdió cuando las muertes de escenas se excluyeron del análisis (OR 0,93; IC del 95\%: 0,58 a 1,46, p=0,78). Además, la probabilidad de muerte en estos pacientes se asoció con el sexo masculino (OR 1,45, $p=0,034)$, el aumento de la edad (OR 1,02, $p<0,001)$ y el aumento de la escala de gravedad de la lesión (OR 1,11, p <0,001).

En el estudio de casos y controles de Vera-Lopez et al. ${ }^{(14)}$ se encontró que no había asociación significativa entre el tiempo de atención pre hospitalaria con la mortalidad (OR 0,99; IC95\% 0,97-1,01; $\mathrm{P}=0,318$ ) sin embargo se encontró una gran asociación significativa a la mortalidad a aquellos pacientes que requirieron atención médica pre hospitalaria (OR 28,30; IC95\% 3,60-222,58; $\mathrm{P}=0,001)$, como en el caso del estudio de Boniface et al. ${ }^{(17)}$ y el de Riyapan et al. ${ }^{(12)}$ donde la utilización del servicio médico de emergencia como medio de transporte pre hospitalario en AT estuvo asociado 2,19 veces más a la mortalidad en emergencia y en la derivación (OR ajustado 2,19; IC 95\% [1,88-2,55]), así mismo aumento significativamente la mortalidad en las primeras 24 horas después del ingreso al servicio de emergencia (OR ajustado 2,31; IC 95\% [1,95$2,73])$. Así mismo se halló que la tasa de mortalidad en los departamentos de emergencias y durante la derivación en pacientes con lesiones graves por AT que requirieron ser transportados por el servicio médico de emergencia fue significativamente mayor que en aquellos que no lo fueron ( $2,00 \%$ vs. $0,78 \%$; $p<0,001)$.

En el cohorte retrospectivo de Byrne et al $^{(9)}$ se halló que las probabilidades de muerte en los centros de trauma con el tiempo pre hospitalario más corto fueron significativamente mayores que en los centros con los tiempos pre hospitalarios totales más largos (odds ratio [OR], 2,00; intervalo de confianza del 95\% [ IC], 1,43$2,78)$. Al final se determinó que no hubo relación entre el tiempo pre hospitalario y la mortalidad global Por lo tanto, no hay evidencia de la inclusión del tiempo pre hospitalario de servicios médicos de emergencia en el análisis de evaluación comparativa del desempeño.

En el estudio de Champion et al. ${ }^{(16)}$ y se analizó 55537 muertes dentro del rango de 5 minutos a 4 horas del accidente, encontrándose que la mayor cantidad de muertes después del accidente se hallaban en el periodo prehospitalario más que en el hospitalario, mostrándose que el riesgo de muerte después del accidente por cada minuto durante los primeros 30 minutos era de $0,4 \%$, que aumento a un $1 \%$ por minuto en los 60 minutos posteriores, que progresivamente fue estabilizándose, por tal motivo ponen énfasis a la respuesta temprana del SME, el sistema de reanimación y la importancia del actuar temprano por el centro de trauma.

\section{DISCUSIÓN}

En la revisión sistemática realizada no de hallaron Meta análisis, por lo que consideramos el presente artículo uno de los primeros publicados abordando este tema, en especial a nivel nacional.

En esta revisión se buscó la asociación entre el tiempo de atención prehospitalaria y la mortalidad en víctimas de AT, que viene siendo la primera prioridad en salud por la elevada mortalidad que los AT representan. Se encontró que si bien el tiempo prolongado de atención prehospitalaria hasta la llegada a un centro de emergencia es un factor importante en la mortalidad y si se muestra una asociación significativa a esta como se muestra en los estudios de Chandrasekharan A et al. ${ }^{(6)}$, GAUSS et al. ${ }^{(5)}$, TANSLEY et al. ${ }^{(15)}$, BONIFACE et al. ${ }^{(17)}$, Champion et al. ${ }^{(16)}$ o Gonzalez et al. ${ }^{(10)}$, no es el único factor determinante de mortalidad, ni el más importante. En diversos estudios se halló una asociación significativa, la presencia de otros factores como el tipo de transporte, la edad, el sexo, el tipo de lesión, la severidad de la lesión, el estado de conciencia, así como el uso de medios de seguridad como cascos o cinturón de seguridad, el consumo de alcohol, los usuarios vulnerables en la carretera, las características del ambiente, si es rural o urbano, así como la distancia entre el lugar del accidente y el centro de emergencia y el uso o no de un sistema de emergencia para transporte prehospitalario, mostrando este en los diversos estudios una significativa asociación a la mortalidad ${ }^{(11)}$ así como una asociación poco significativa en otros estudios $^{(9,10)}$, pudiendo deberse esta variación a la severidad del trauma que presenta el paciente, siendo este el factor más importante y constantemente asociado significativamente a una mayor mortalidad.

Es de esperarse que el resultado del transporte por un servicio médico de emergencias más calificado sea más eficaz debido al manejo por personal preparado para intervenir en esta clase de emergencias con un manejo seguro y adecuado, con la aplicación de equipos de protección para prevenir lesiones 
adicionales o empeoramiento súbito del estado actual, así como un soporte vital especializado para atención prehospitalaria tales como el control de hemorragias, vía respiratoria y la velocidad de llegada desde la llamada al SME hasta la llegada de la víctima a un centro de atención, aun cuando hay estudios como el de Vera-López et al. ${ }^{(14)}$ que asocian la prestación de servicios prehospitalarios en víctimas de accidentes de tránsito a una mayor mortalidad y aun así no encontrando asociación entre un largo tiempo de respuesta prehospitalaria y esta, se debe a que en su mayoría, como lo demuestran en el estudio de Lovely et al. ${ }^{(11)}$, existen otros factores, como los antes mencionados, que se asocian a una mayor mortalidad, como es el índice de severidad de la lesión.

Sin embargo no es el único motivo asociado a la mortalidad hospitalaria, siendo el nivel del centro de atención y su capacidad de actuar frente a esta clase de emergencias y lesiones graves un factor importante, encontrándose en el estudio de Mills et al.7 que existe una asociación a la mortalidad más baja en centros hospitalarios no terciarios que en centros hospitalarios terciarios, siendo la capacidad del centro de salud donde sea llevado un factor importante en la mortalidad de las víctimas de AT.

Aunque hayan estudios donde no se encuentre una asociación entre el tiempo de atención pre hospitalaria y la mortalidad en víctimas por AT, hay que tener en cuenta que la atención pre hospitalaria depende también de otros factores, en una revisión sistemática Harmsen et al. ${ }^{(4)}$ se encontró que el transporte rápido parece beneficioso para pacientes que sufren traumatismos graves con compromiso de la presión arterial, más en pacientes con lesiones más leves y hemodinámicamente estables, no hay mayor aumento de la mortalidad respecto al tiempo de atención pre hospitalaria.

Un aspecto a considerar es la limitada disponibilidad de estudios que contribuyan a la asociación de estas variables a nivel nacional, siendo necesario de fortalecer las investigaciones en esta área.

\section{CONCLUSIÓN}

El tiempo de atención pre hospitalaria en AT se encuentra significativamente asociada a la mortalidad hospitalaria de los pacientes por la necesidad de la precoz estabilización del cuadro del paciente. Sin embargo esto depende de diversos factores propios del accidente, y la gravedad del paciente en la escena, la adecuada extracción de la victima de AT de la escena que puede requerir mayor tiempo que el previsto debido a las limitaciones del servicio que otorga atención o por la dificultad del procedimiento, por tal motivo algunos estudios mostraban menor mortalidad en tiempos pre hospitalarios más largos. Dados los resultados se propone estudiar factores asociados al largo tiempo de atención pre hospitalaria.

Agradecimiento: Al dr. Jhony A. De La Cruz-Vargas por su paciencia y el aporte como supervisor y revisor del presente artículo.

Contribuciones de autoría: MJGFS y BJVZ realizaron la revisión sistemática, la concepción del artículo y la redacción del artículo. JADLCV realizó la revisión crítica del artículo y la aprobación de la versión final.

Financiamiento: Autofinanciado.

Conflicto de interés: Los autores declaran no tener conflictos de interés. El presente trabajo fue presentado en el Taller de Elaboración de Tesis de la Facultad de Medicina Humana de la UNIVERSIDAD RICARDO PALMA.

Recibido: 09 de diciembre del 2019

Aprobado: 27 de diciembre del 2019

Correspondencia: María Jesús de Guadalupe Fernandez Sandoval. Dirección: Av. Los Gorriones 365, La campiña-Chorrillos, Lima-Perú. Teléfono: (+51) 935859812

Correo:majus_1996@hotmail.com 


\section{REFERENCIAS BIBLIOGRÁFICAS}

1. Peden $M$, Scurfield $R$, Sleet $D$, Mohan $D$, Hyder $A A$, Jarawan $E$ y Mathers $C$ (eds). Informe mundial sobre prevención de los traumatismos causados por el tránsito Organización Mundial de la Salud. Washington: OMS; 2004. (https:// www.who.int/violence_injury_prevention/publications/road_traffic/world_ report/summary_es.pdf)

2. (Consejo Nacional de Seguridad Vial. (2011). Estadísticas de accidentes de tránsito. Recuperado el 20 de marzo de 2013, de http://www.mtc.gob.pe/ cnsv/estadísticas.)

3. Bvs.minsa.gob.pe. (2009). ACCIDENTES DE TRÁNSITO PROBLEMA DE SALUD PÚBLICA - Informe Nacional. [online] Available at: http://bvs.minsa.gob.pe/ local/MINSA/829_MINSA1412.pdf [Accessed 31 Aug. 2019].

4. Harmsen AMK, Giannakopoulos GF, Moerbeek PR, Jansma EP, Bonjer HJ, Bloemers FW. The influence of prehospital time on trauma patients outcome: A systematic review. Injury. abril de 2015;46(4):602-9. Disponible en: https:// doi.org/10.1016/j.injury.2015.01.008

5. Tohme S, Delhumeau C, Zuercher M, Haller G, Walder B. Prehospital risk factors of mortality and impaired consciousness after severe traumatic brain injury: an epidemiological study. Scand J Trauma Resusc Emerg Med. 2014;22(1):1.Disponible en: https://dx.doi.org/10.1186\%2F1757-7241-22-1

6. Chandrasekharan A, Nanavati AJ, Prabhakar S, Prabhakar S. Factors Impacting Mortality in the Pre-Hospital Period After Road Traffic Accidents in Urban India. Trauma Mon [Internet]. 26 de mayo de 2016 [citado 18 de octubre de 2019];21(3). Disponible en: https://doi.org/10.5812/traumamon.22456

7. Mills EHA, Aasbjerg K, Hansen SM, Ringgren KB, Dahl M, Rasmussen BS et al. Prehospital time and mortality in patients requiring a highest priority emergency medical response: a Danish registry-based cohort study. BM Open. noviembre de 2019:9(11):e023049. Disponible en: https://doi. org/10.1136/bmjopen-2018-023049

8. Brown E, Tohira H, Bailey P, Fatovich D, Pereira G, Finn J. Longer Prehospita Time was not Associated with Mortality in Major Trauma: A Retrospective Cohort Study. Prehospital Emergency Care. 4 de julio de 2019;23(4):527-37. Disponible en: https://doi.org/10.1080/10903127.2018.1551451

9. Byrne JP, Mann NC, Hoeft CJ, Buick J, Karanicolas P, Rizoli S, et al. The impact of short prehospital times on trauma center performance benchmarking: An ecologic study. Journal of Trauma and Acute Care Surgery. abril de 2016;80(4):586-96. Disponible en: https://doi.org/10.1097/ TA.0000000000000974
10. Gonzalez RP, Cummings GR, Phelan HA, Mulekar MS, Rodning CB. Does increased emergency medical services prehospital time affect patient mortality in rural motor vehicle crashes? A statewide analysis. The American Journal of Surgery. enero de 2009;197(1):30-4. Disponible en: https://doi. org/10.1016/j.amjsurg.2007.11.018

11. Lovely R, Trecartin A, Ologun G, Johnston A, Svintozelskiy S, Vermeylen F, et al. Injury Severity Score alone predicts mortality when compared to EMS scene time and transport time for motor vehicle trauma patients who arrive alive to hospital. Traffic Injury Prevention. 28 de diciembre de 2018;19(sup2):S167-8. Disponible en: https://doi.org/10.1080/15389588.2018.1532217

12. Riyapan S, Thitichai P, Chaisirin W, Nakornchai T, Chakorn T. Outcomes of Emergency Medical Service Usage in Severe Road Traffic Injury during Tha Holidays. WestJEM. 5 de marzo de 2018;19(2):266-75. Disponible en: https:// doi.org/10.5811/westjem.2017.11.35169

13. Gauss T, Ageron F-X, Devaud M-L, Debaty G, Travers S, Garrigue D, et al. Association of Prehospital Time to In-Hospital Trauma Mortality in a Physician-Staffed Emergency Medicine System. JAMA Surg [Internet]. 25 de septiembre de 2019 [citado 18 de octubre de 2019]; Disponible en: https://doi. org/10.1001/jamasurg.2019.3475

14. Vera-López JD, Pérez-Núñez R, Gómez-García L, Hidalgo-Solórzano E, Fraga-Sastrías JM. La respuesta del Sistema Médico de Emergencias y su relación con distintos resultados en salud en personas lesionadas por el tránsito de dos ciudades mexicanas. Cad Saúde Pública [Internet]. 22 de octubre de 2018 [citado 18 de octubre de 2019];34(10). Disponible en: http:// dx.doi.org/10.1590/0102-311x00144916

15. Tansley G, Schuurman N, Bowes M, Erdogan M, Green R, Asbridge M, et al. Effect of predicted travel time to trauma care on mortality in major trauma patients in Nova Scotia. cjs. 1 de abril de 2019;62(2):123-30. Disponible en:

https://doi.org/10.1503/cjs.004218

16. Champion HR, Lombardo LV, Wade CE, Kalin EJ, Lawnick MM, Holcomb JB. Time and place of death from automobile crashes: Research endpoint implications. Journal of Trauma and Acute Care Surgery. septiembre de 2016;81(3):420-6. Disponible en: https://doi.org/10.1097/ TA.0000000000001124

17. Boniface R, Museru L, Munthali V, Lett R. INJURY EXPERIENCE INTANZANIANEED FOR INTERVENTION. East African Medical Journal. 2013;9. Disponible en https://www.ncbi.nlm.nih.gov/pubmed/26859008

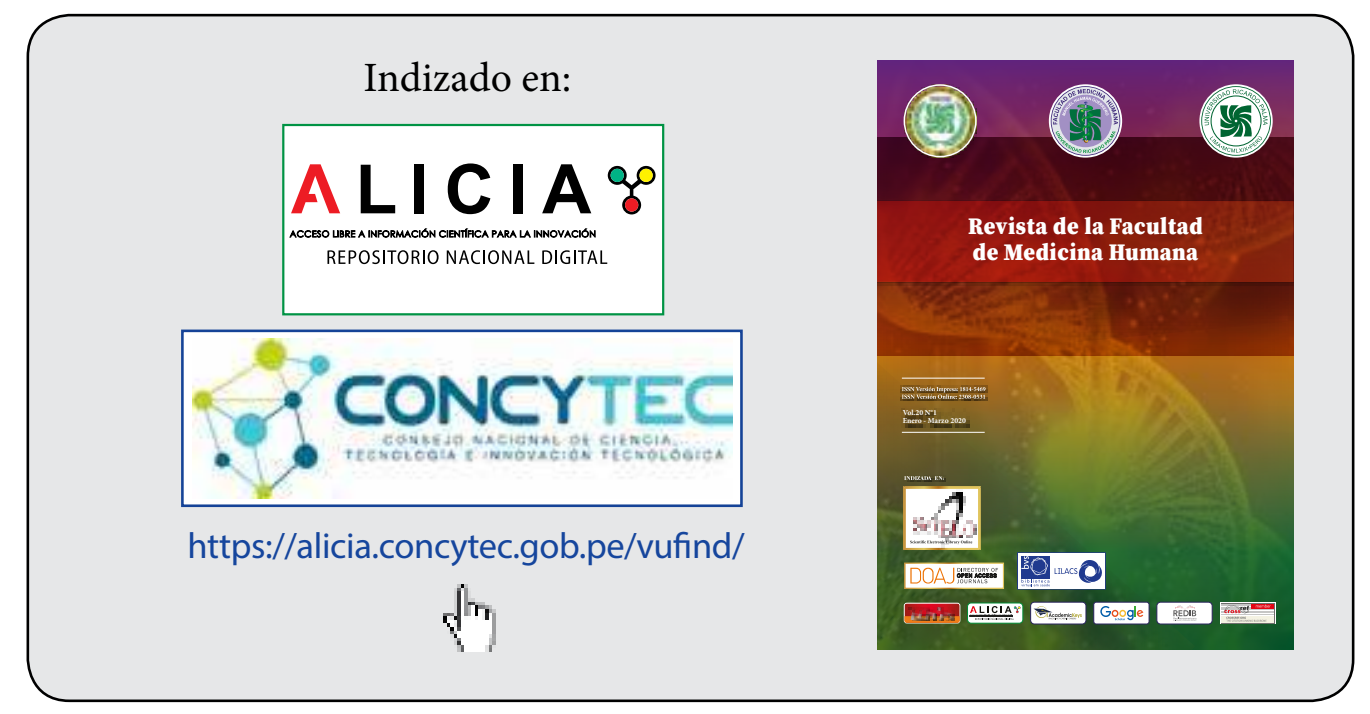

